\title{
Mapping 3D X-ray Fluorescence Datasets to Elemental Distributions using Principal Component Analysis and Fitting ${ }^{[5]}$
}

\author{
Stefan Vogt*, Chris Jacobsen**, Ann LeFurgey***, David Kopf ****, Barry Lai*, Peter \\ Ingram***, Jörg Maser* \\ * Experimental Facilities Division, Argonne National Laboratory, 9700 S Cass Ave, Argonne, IL \\ 60439, USA \\ ** Department of Physics and Astronomy, Stony Brook University, Stony Brook, NY 11794, USA \\ *** Duke University Medical Center and VA Medical Center, Durham, NC, USA \\ **** DAKX, LLC, P.O.Box 1758, Pittsboro, NC 27312
}

X-ray-induced X-ray fluorescence (XRF) scanning microscopy is a powerful technique to map and quantify element distributions in biological specimens, such as cells and bacteria. Ideally full spectra are acquired and saved at each scan position to allow a later deconvolution of overlapping peaks and removal of the background. Per-pixel dwell times are chosen to compromise between high spatial resolution (corresponding to many sampling points) and good counting statistics in order to achieve reasonable scan times.

Several approaches are possible to map the fluorescence counts detected by the energy dispersive detector to quantify element concentrations. Spectral filtering based on spectral region of interests (ROIs) is the simplest and fastest method, but lacks precision when there is interference by other peaks or by overall background. Fitting of the single spectra at every pixel can account much better or these interferences, but can be a slow process as many spectra need to be fit. For example, in a 100x100 pixel scan, fits of 10000 spectra need to be calculated and potentially inspected. Also, as the photon statistics in a single pixel spectrum are low, the number of fit parameters has to be limited.

Principal component analysis (PCA) provides a method to correlate an XRF dataset with full spectra at each scan point and to weigh each component of the spectrum, and its corresponding eigenimage, according to its respective significance in the dataset [1-3]. In particular, photon noise is not correlated among pixels and therefore does not contribute to the principal components. We show that, by fitting the eigenspectra of the principal components, one can then generate maps of fitted elemental components with high accuracy without the need to fit the spectra of single pixels. This drastically reduces the number of spectra that need to be fit, typically to less than 20 . Additionally, the correlation of elemental distributions can be used to reveal information about the number and composition of the different major constituents of a cell.

Using this technique, we have analyzed the elemental distribution in Leishmania donovani parasites that were treated with a therapeutic drug containing antimony (Pentostam, The Wellcome Foundation, London, UK). The Sb L-lines overlap significantly with the Ca K $\alpha$-lines (see Fig. 1), so that the naturally occurring $\mathrm{Ca}$ content of $L$. donovani makes an accurate analysis and mapping of the $\mathrm{Sb}$ difficult. We will show examples of the PCA-based fitting and compare it both to spectral ROIs and per-pixel fitting approaches (see, e.g., Fig. 1). 
References

[1] Malinowski, E. R., Factor Analysis in Chemistry (J. Wiley, New York, 1991).

[2] Osanna, A. and Jacobsen, C., "Principal Component Analysis for Soft X-ray Spectromicroscopy," in X-ray Microscopy, AIP Conference Proceedings 507 (American Institute of Physics, Melville, 2000), pp. 350-355.

[3] Vogt, S., Maser, J., and Jacobsen, C., "Data Analysis for X-ray Fluorescence Imaging," Journal de Physique IV 104, 617-622, 2003.

[4] LeFurgey, A., Gannon, M., Blum, J., and Ingram, P. “Leishmania donovani amastigotes mobilize organic and inorganic osmolytes during regulatory volume decrease," J. Eukaryotic Microbiology, in press.

[5] The work at Argonne is supported by the U.S. Department of Energy, Office of Basic Energy Sciences, under Contract No. W-31-109-ENG-38. The Leishmania donovani project is supported by a VA Merit Award to AL.
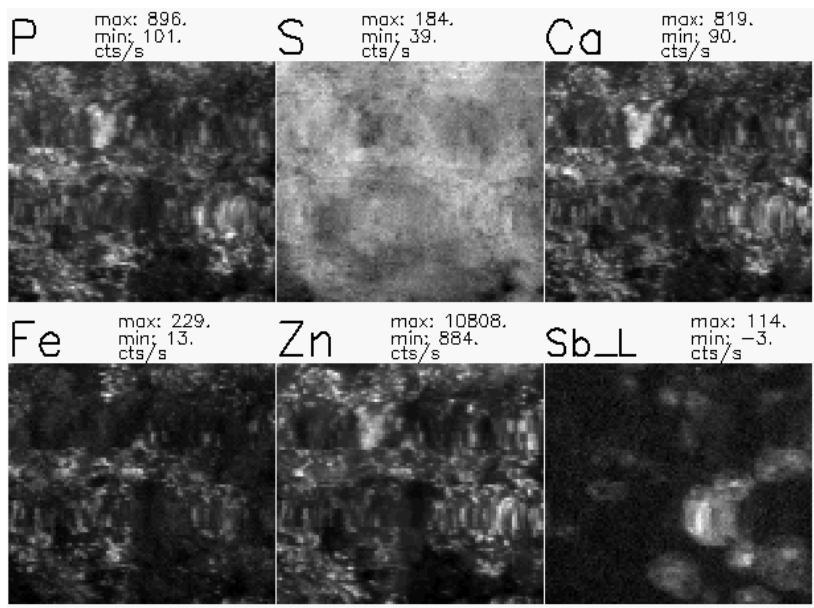

$10 \mathrm{~nm}$
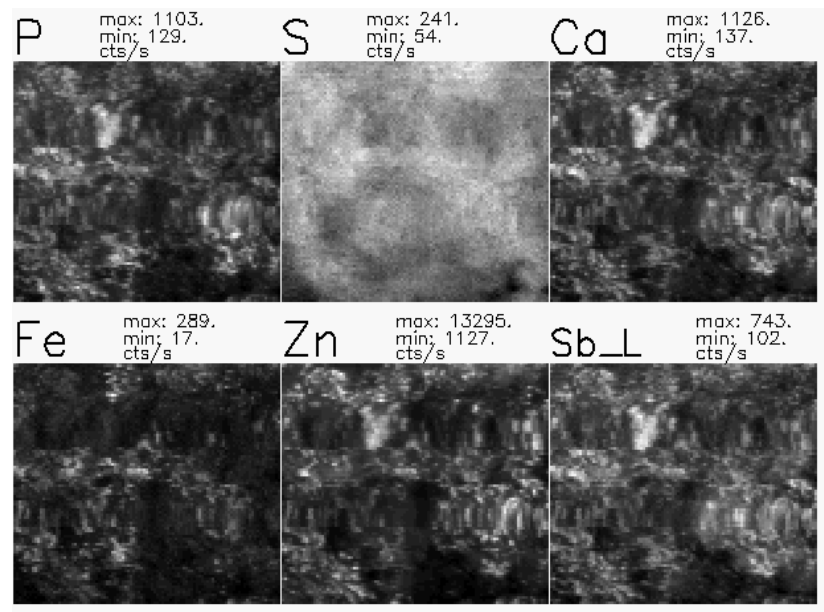

$10 \mathrm{~nm}$

FIG. 1. Maps of selected elements of a cluster of several Leishmania donovani amastigote forms [4], generated from a full 3D (x,y, energy) X-ray fluorescence dataset. On the left-hand side, the maps were calculated using principal component analysis and fitting. The $\mathrm{Sb}$ distribution is quite distinct from the other elements and shows that the $\mathrm{Sb}$ content of different L. donovani cells varies significantly. By contrast, the maps on the right hand side were generated using only spectral filtering (ROIs). The signal in the $\mathrm{Sb} \mathrm{L}_{\alpha, \beta}$ map is dominated by bleed-through from the $\mathrm{Ca} \mathrm{K}_{\alpha, \beta}-$ lines, and does not show the true $\mathrm{Sb}$ distribution. 